\title{
On Fostering a Culture of
}

Research Cyberinfrastructure Grant Proposals within a

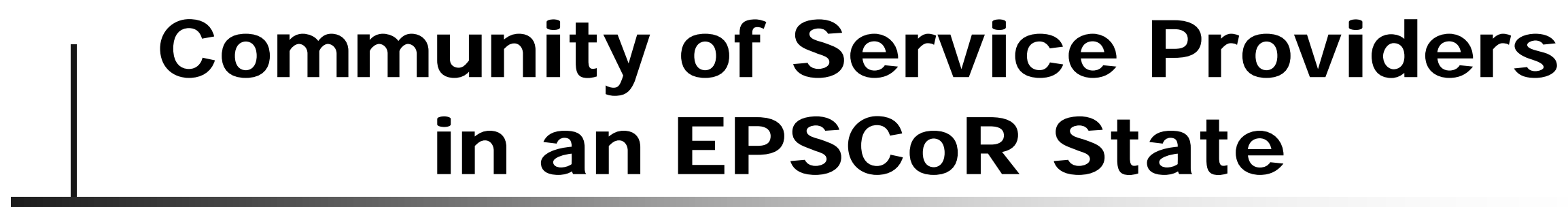

Henry Neeman, University of Oklahoma

Kate Adams, Great Plains Network

Joshua Alexander, University of Oklahoma

Dana Brunson, Oklahoma State University

S. Patrick Calhoun, University of Oklahoma

James Deaton, OneNet

Franklin Fondjo Fotou, Langston University

Karl Frinkle, Southeastern Oklahoma State University

Zane Gray, University of Oklahoma

Evan Lemley, University of Central Oklahoma

George Louthan, Oklahoma Innovation Institute

Greg Monaco, Great Plains Network

Mike Morris, Southeastern Oklahoma State University

Joel Snow, Langston University

Brett Zimmerman, University of Oklahoma

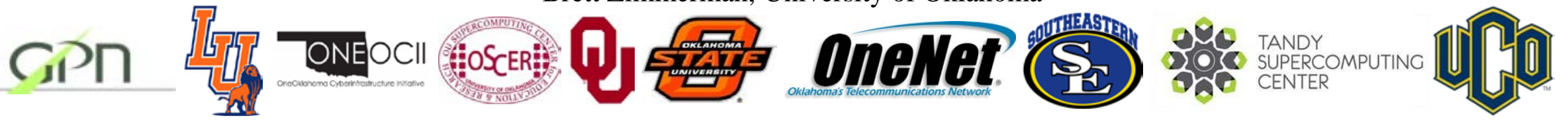




\section{OneOklahoma \\ Cyberinfrastructure Initiative}
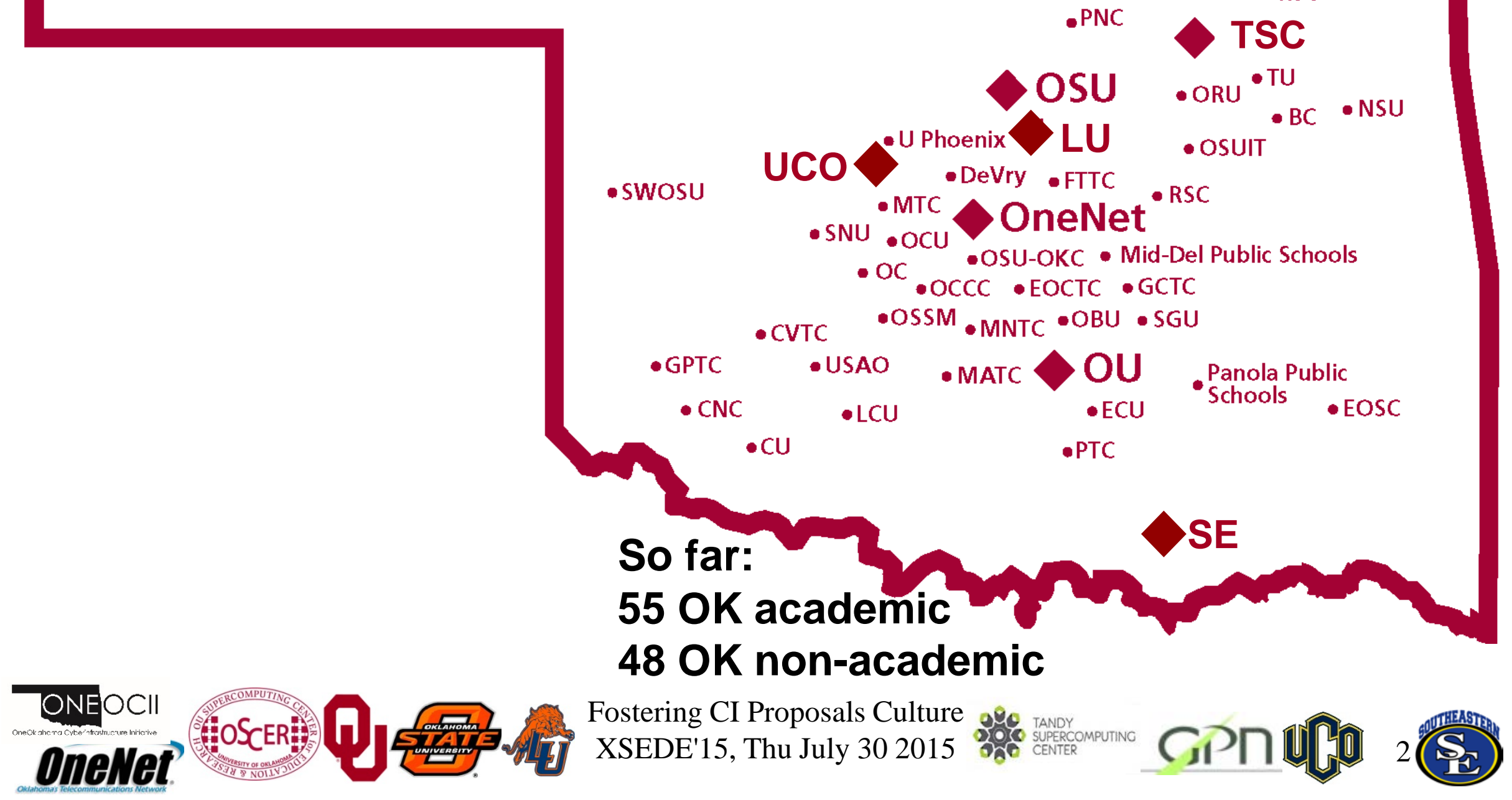


\section{Proposal Summary}

- 2001-2014 CI

- 2001: Minimal CI Resources

- 2014 - 200 teraflops and emerging shared DMZ

- Expansion in 2015: OU's new cluster arriving now

- 2008-present: 14 CI proposals

- NSF MRI: OU, OSU, Langston U, UCO: 6 submits, 4 awards

- NSF EPSCoR RII Track-2 (CI focus) with KS: 1 submit, 1 award

- NSF EPSCoR RII C2: 1 submit, 1 award

- NSF CC-NIE: 1 submit, 1 award

- NSF CC*IIE: 3 submits, 2 awards

- NSF CC*DNI: 2 submits (both pending)

- 12 reviewed, 10 awarded (83\%) / 2 declined

- OK FY 2014 NS average - 20\% 


\section{Capacity}

- 2002: 1.2 TFLOPs statewide, 1 Service Provider

- 2005: 6.5 TFLOPs statewide, 1 Service Provider

- 2008: 40 TFLOPs statewide, 2 Service Providers

- 2012: 200+ TFLOPs statewide, 4 Service Providers

- 2015: 400+ TFLOPs statewide, 5 Service Providers 


\section{OneOklahoma}

Cyberinfrastructure

Initiative 


\section{OCII vs OneOCII}

- Originally OCII, established under Oklahoma’s 2008-13 NSF EPSCoR Research Infrastructure Improvement (RII) Track-1 grant.

- Became OneOCII under Oklahoma’s 2013-18 RII Track-1.

- State Science \& Technology plan, a required proposal component for RII Track-1, was the OneOklahoma Science \& Technology Plan. 


\section{OneOCII Goals}

- Reach institutions outside the mainstream of advanced computing.

- Serve every higher education institution in Oklahoma that has relevant curricula.

- Educate Oklahomans about advanced computing.

- Attract underrepresented populations and institution types into advanced computing. 


\section{OneOCII Service Methodologies Part 1}

- Access: to supercomputers and related technologies

(20 OK academic institutions to date).

- Dissemination: Oklahoma Supercomputing Symposium annual advanced computing conference has reached 112 academic institutions, 143 commercial, 36 government, 20 nongovernmental (25 OK academic institutions to date).

- Education: "Supercomputing in Plain English” (SiPE) workshop series: 11 talks about advanced computing, taught with stories, analogies and play rather than deep technical jargon. Have reached 362 institutions (academic, government, industry, nonprofit) in 51 US states and territories and 17 other countries (16 OK academic institutions to date). 


\section{OneOCII Service Methodologies Part 2}

- Faculty/Staff Development: Workshops held at OU and OSU on advanced computing and computational science topics, sponsored by the National Computational Science Institute, the SC supercomputing conference series, the Linux Clusters Institute, the Virtual School for Computational Science \& Engineering. Oklahoma is the only state to have hosted multiple events sponsored by each of these (18 OK academic to date).

- Informatics: research facilitators embedded in specific research projects (and largely funded by them)

- Outreach: "Supercomputing in Plain English" (SiPE) overview talk (25 OK academic to date). 


\section{OneOCII Service Methodologies Part 3}

- Proposal Support: Letters of commitment for access to OneOCII resources; collaborations with OneOCII lead institutions (4 OK academic, 1 nongovernmental).

- Stewardship: Research data stewardship initiative, led by Libraries.

- Technology: Got or helped get technology (e.g., network upgrade, mini-supercomputer, hi def video camera for telepresence) for that institution (14 OK academic to date). 


\section{OneOCII Service Methodologies Part 4}

- Workforce Development - (39 OK academic)

- Oklahoma Information Technology Mentorship Program (OITMP)

- "A Day in the Life of an IT Professional” presentations to courses across the full spectrum of higher education.

- Job shadowing opportunities and direct mentoring of individual students.

- Institution Types: high schools, career techs, community colleges, regional universities, PhD-granting universities.

- Special effort to reach underrepresented populations: underrepresented minorities, non-PhD-granting, rural 


\section{OneOCII Institution Profile}

To date, OneOCII has served 103 Oklahoma institutions, agencies and organizations:

- 55 OK academic

- 48 OK non-academic

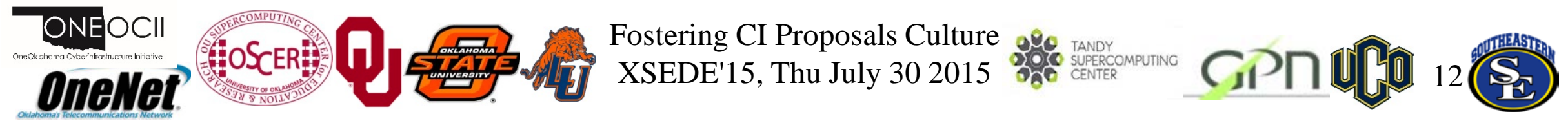




\section{OneOCII Institution Profile}

To date, OneOCII has served 103 Oklahoma institutions, agencies and organizations:

- 55 OK academic

- Universities \& Colleges

- 3 comprehensive PhD-granting

- 20 regional non-PhD-granting

- Community Colleges: 10

- Career techs: 14

- Secondary schools: 4

- Public school systems: 4

- 48 OK non-academic 


\section{OneOCII Institution Profile}

So far, OneOCII has served:

- 55 OK academic

- 10 Minority Serving Institutions

- 16 other institutions with above state average and national average for one or more underrepresented minorities

- 48 OK non-academic
Minority Serving Institutions

- Oklahoma’s only Historically Black College or University

- Langston U (Langston)

- Native American Serving Non-tribal Institutions

- East Central U (Ada)

- Northeastern Oklahoma A\&M College (Miami)

- Northeastern State U (Tahlequah)

- Southeastern Oklahoma State U (Durant)

- Tribal Institutions

- College of the Muscogee Nation (Okmulgee)

- Comanche Nation College (Lawton)

- Pawnee Nation College (Pawnee)

- Sequoyah High School

- Other Minority Serving Insitution

- Bacone College (Muskogee) 


\section{OneOCII Institution Profile}

To date, OneOCII has served 103 Oklahoma institutions, agencies and organizations:

- 55 OK academic institutions

- 48 OK non-academic organizations

- 16 commercial

- 19 government

- 2 military

- 11 non-governmental 


\section{OneOCII Outcomes: Research}

External research funding to OK institutions facilitated by OneOCII lead institutions (Fall 2001- Summer 2013): \$165M+

- Funded projects facilitated: over 200

- OK faculty and staff: over $\mathbf{1 0 0}$ in 20 academic disciplines

- Specifically needed OneOCII just to be funded: over \$42M (necessary but far from sufficient)

- NSF EPSCoR RII Track-1 (2008-13): \$15M to OK

- NSF EPSCoR RII Track-1 (2013-18): \$20M to OK (+\$4M State Regents)

- NSF EPSCoR RII Track-2: \$3M to OK

- NSF EPSCoR RII C2: $\$ 1.17 \mathrm{M}$ to OK

- NSF MRI (OU): $\$ 793 \mathrm{~K}$

- NSF MRI (OSU): $\$ 908 \mathrm{~K}$

- NSF MRI (Langston U): \$250K

- NSF MRI (UCO): $\$ 304 \mathrm{~K}$

- NSF CC-NIE (OU/OSU/LU/TSC): \$500K

- NSF CC*IIE (OU): $\$ 400 \mathrm{~K}$

- NSF CC*IIE (OneNet+GPN): \$350K 


\section{OneOCII Outcomes: Education}

\section{Teaching: 9 institutions including 3 MSIs}

- Taught parallel computing using OneOCII resources:

- Cameron U - multiple times

- East Central U (NASNI)

- Oklahoma City U - multiple times

- Taught parallel computing via LittleFe baby supercomputer and OneOCII resources:

- Southeastern Oklahoma State U (NASNI) - 3 semester sequence, multiple times

- Taught computational chemistry using OneOCII resources:

- Northeastern State U (NASNI) - multiple times

- Southern Nazarene U

- Rogers State U - multiple times

- Taught Bioinformatics using OneOCII resources:

- $\underline{\text { U Tulsa }}-2$ semester sequence 


\section{OneOCII Outcomes: Resources}

6 institutions including 2 MSIs, plus C2 institutions

- NSF Major Research Instrumentation grants: \$1.95M

- OU: Oklahoma PetaStore, \$793K (in production)

- Oklahoma State U: Cowboy cluster, \$909K (in production)

- Langston U: cluster, \$250K (in production)

- U Central Oklahoma: cluster, \$304K (being deployed)

- LittleFe baby supercomputer grants (\$2520 each)

- OU: Ron Barnes

- Oklahoma City U: Larry Sells \& John Goulden

- Southeastern Oklahoma State U: Mike Morris \& Karl Frinkle

- Networking

- NSF EPSCoR RII C2 grant: \$1.17M

- NSF CC-NIE grant: $\$ 500 \mathrm{~K}$

- NSF CC*IIE grant: $\$ 400 \mathrm{~K}$ 


\section{University of Oklahoma}




\section{University of Oklahoma}

- NSF MRI 2003-6 (OU, LU): Itanium2 cluster (\$340K)

- DOE EPSCoR 2004-11 (OU, OSU, LU): Established Oklahoma Center for High Energy Physics (\$2.6M)

- NSF EPSCoR RII Track-1 2008-13 (OU, OSU, Samuel Roberts Noble Foundation): Established OCII, co-led by OU and OSU $(\$ 15 \mathrm{M})$.

- NSF EPSCoR RII Track-2 2009-13 (OU, OSU, KU, KSU): Established Informatics team, created software CI (\$6M).

- NSF EPSCoR RII Cyber Connectivity (OU, OSU, LU, OneNet, Noble, U Tulsa): Statewide research ring network and institutional CI had network upgrades. 


\section{University of Oklahoma}

- NSF Major Research Instrumentation 2010-14 (OU): Oklahoma PetaStore.

- NSF EPSCoR RII Track-1 2013-18 (OU, OSU, Noble): solidified Informatics, added Data Stewardship, transitioned OCII to OneOCII, led to weekly OneOCII conference calls.

- NSF CC-NIE 2013-15 (OU, OSU, LU, OneNet, Oklahoma Innovation Institute/Tandy Supercomputing Center; adding U Central Oklahoma): OneOklahoma Friction Free Network.

- NSF CC*IIE 2014-16 (OU): ACI-REF (including ACI-REF Virtual Residency program). 


\section{Oklahoma}

\section{State University}




\section{Oklahoma State U}

- November, 2007

- 19 users

- Hired yours truly.

- Met Henry Neeman, formed OCII

- By 2010,

- Had over 150 users

- Modestly more resources and needed

- More resources

- More staff
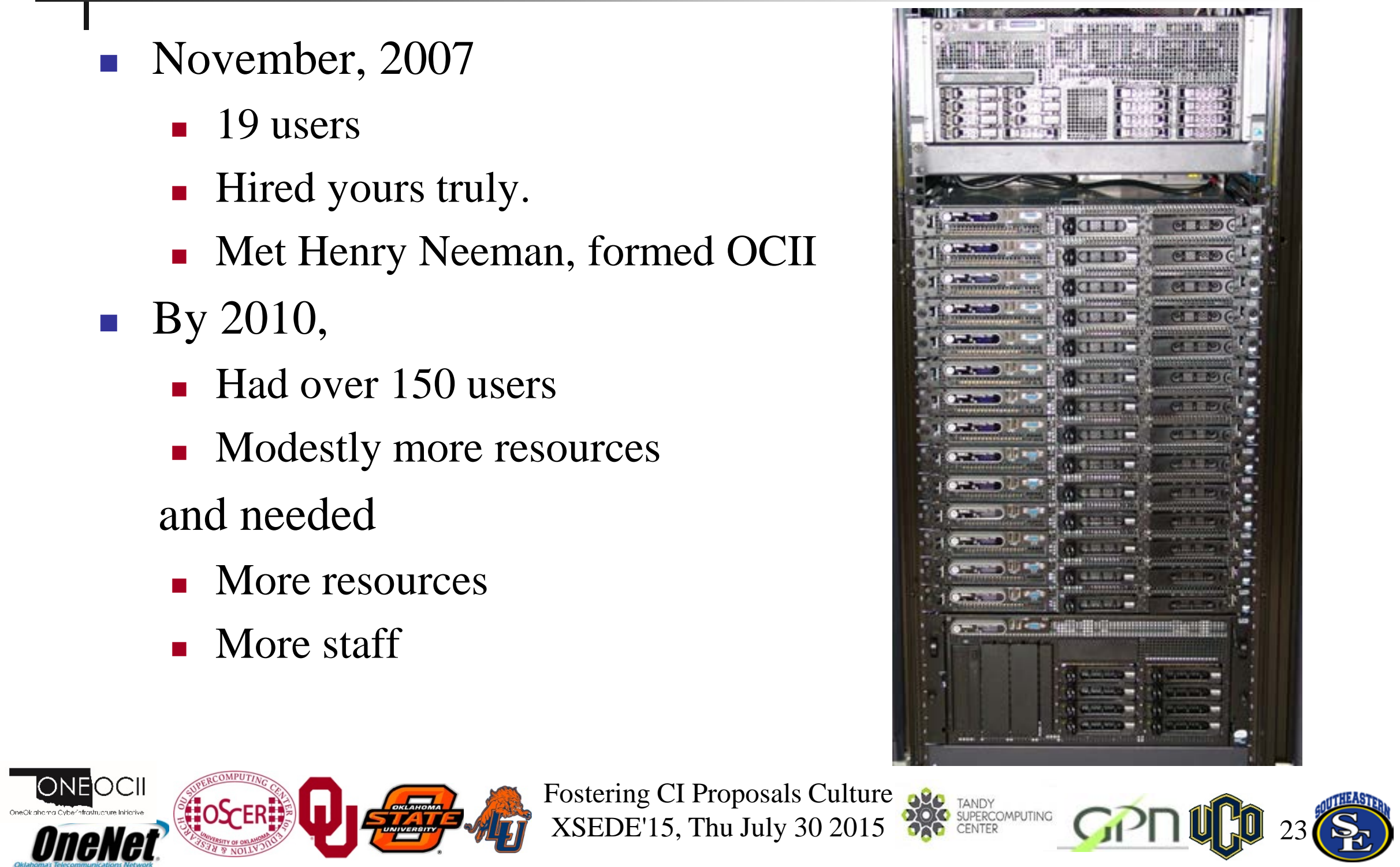


\section{Oklahoma State U}

- Community ideas to the rescue?

- Campus researchers

- Oklahoma Cyberinfrastructure Initiative

- Great Plains Network

- XSEDE Campus Champions

- NSF MRI proposal (first time as PI; Henry talked me into writing it!)

- Mentorship from Henry - especially in articulating the vision to stakeholders and proposal writing. 


\section{Oklahoma State U}

- Cowboy is funded by an NSF grant for $\$ 908,812$.

- Cost share funded new full-time position.

- Cowboy is the largest externally funded supercomputer in state history!

- 9x the capacity of the previous cluster.
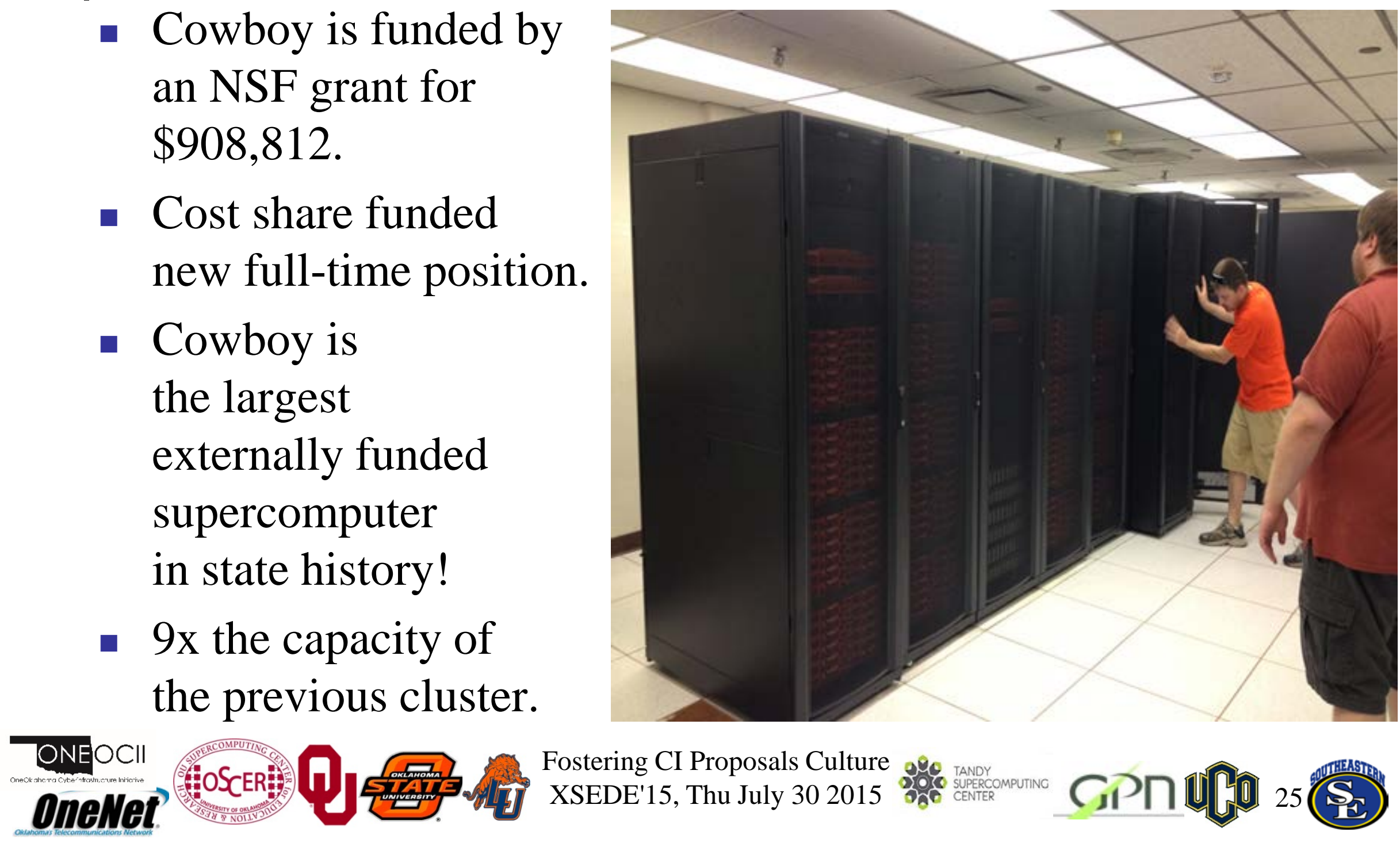


\section{Oklahoma State U}

TODAY:

- New Cloud resource (helped by OneOCII expertise)

- New "Research Cyberinfrastructure Analyst"

- Another new FTE for Program Coordination

- And another FTE Bioinformatics Specialist

- Summer Graduate Research Assistant From one FTE to 5.5 FTEs in 3.5 years. Submitted NSF MRI in 2015 for new cluster. 


\section{Langston \\ University}




\section{Langston U}

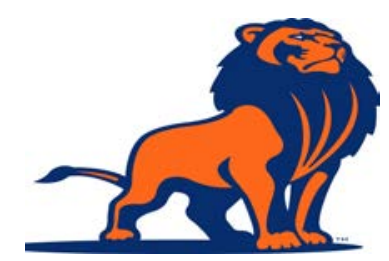

Langston University (LU) is a land grant institution founded in 1897.

- LU is Oklahoma's only Historically Black College/University (HBCU).

- LU has a more diverse student body than at its founding.

- LU is a Primarily Undergraduate Institution (with few advanced degrees conferred).

- Faculty engage in research activities in physics, biology, chemistry, agriculture, and applied mathematics. 


\section{Langston MRI}

- LU's NSF MRI project (“Acquisition of a High

Performance Computing Cluster for Research and

Education,” NSF ACI-1229107, \$250K, 2013-2016, PI

Joel Snow, Co-PI Franklin Fondjo Fotou).

- This funding was critical in deploying "Lucille" at the Langston University Computing Center for Research and Education (LUCCRE) and have enable large scale HPC and HTC on campus. 


\section{Lucille}
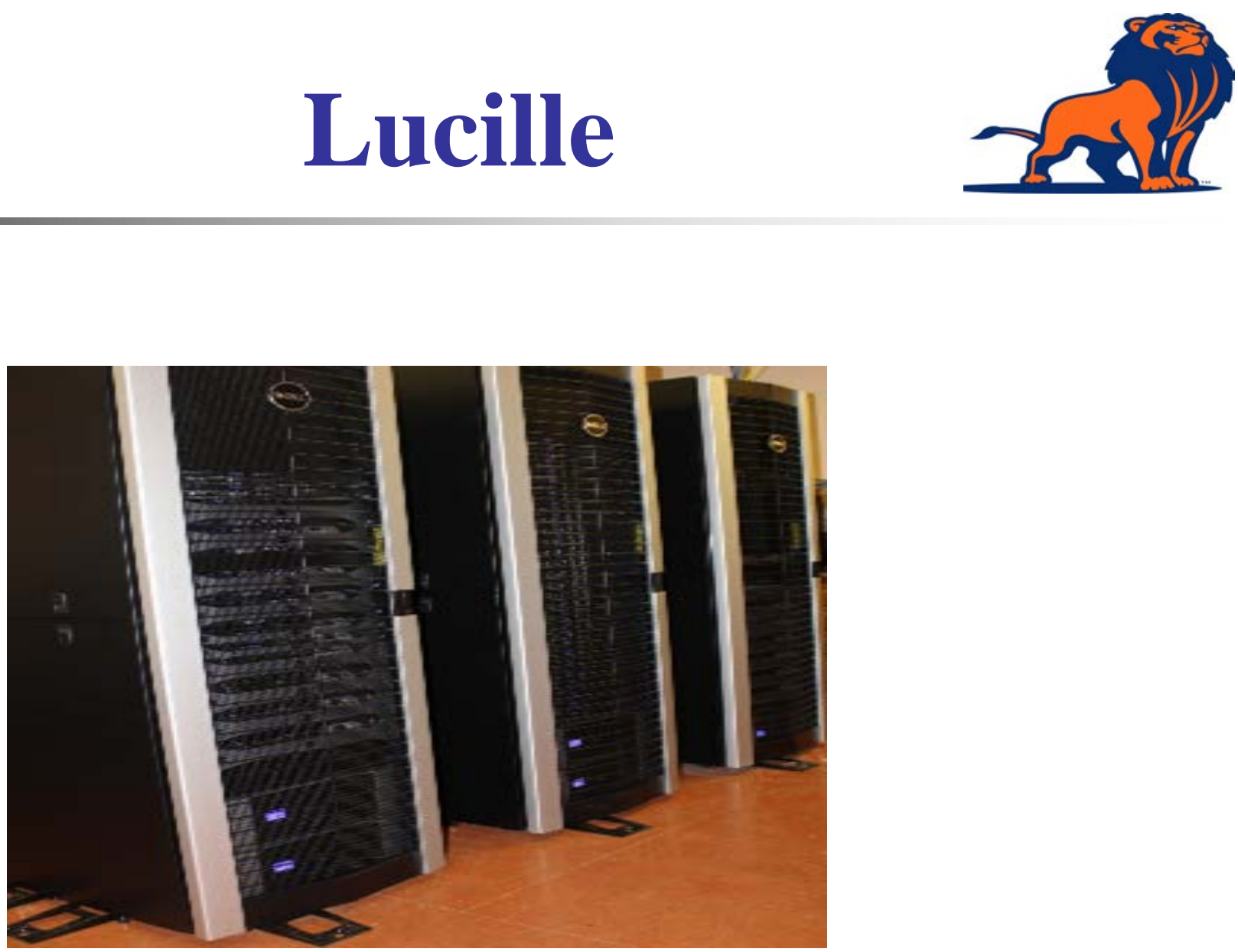

Lucille is a Dell Cluster with 8 TFLOPs CPU, 18.72 TFLOPs GPU power by Rocks/Centos 6 OS. 


\section{Lucille Ganglia}

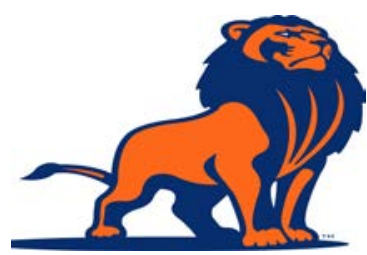

\section{Lucille Ganglia Load July 28, 2015}

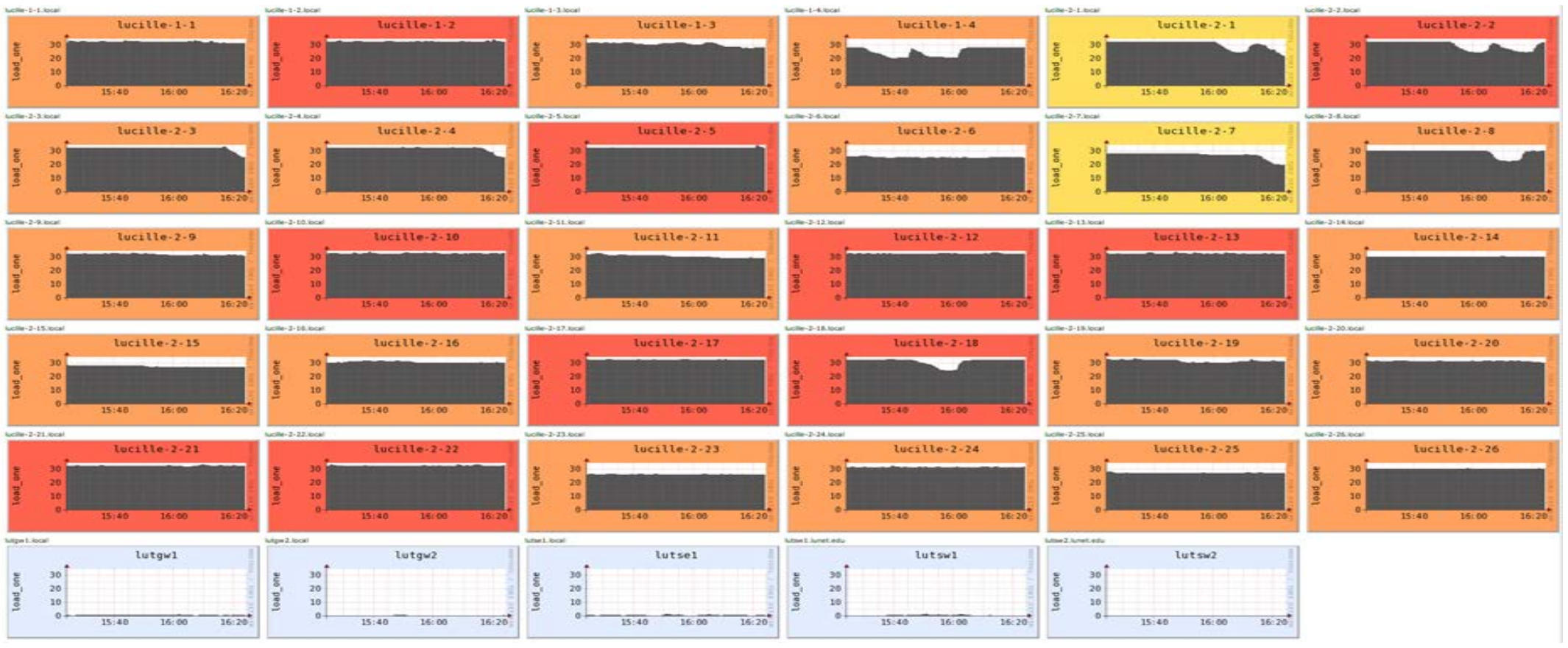

\footnotetext{
ONEOCII

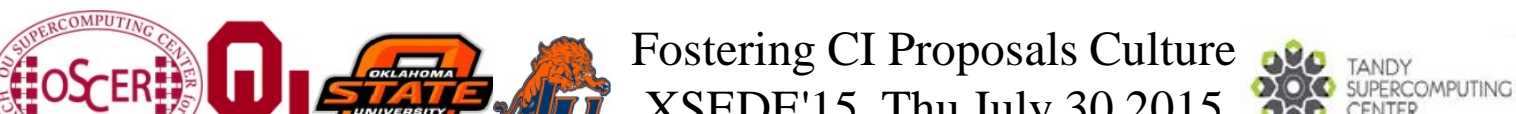




\section{Langston U}

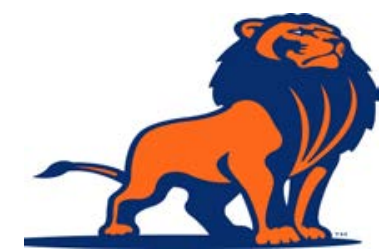

CondorQs at LUCILLE

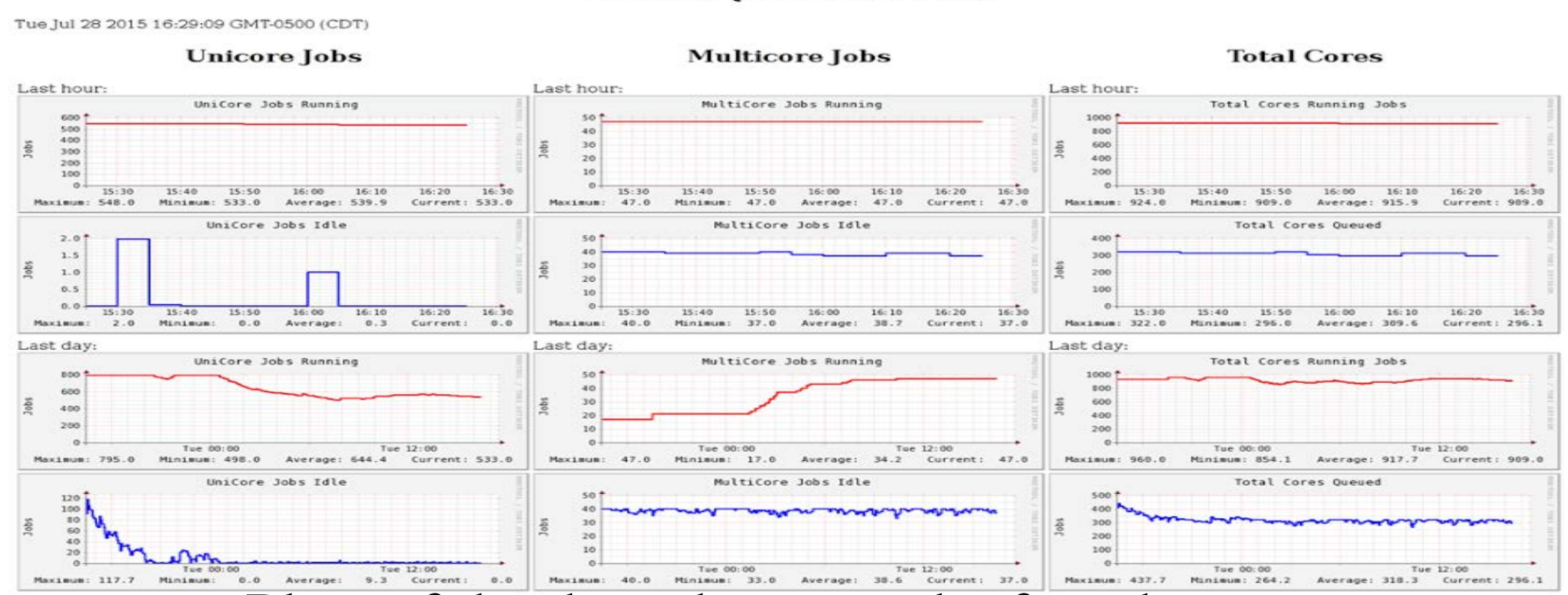

Plots of the data above can be found at:

http://lutgw1.lunet.edu/prod/proddata.html

Links to other Lucille metrics can be found at:

http://lutgw1.lunet.edu/atwork.html

, 


\section{Lucille's Constituency בnd}

- Lucille serves local and distributed users doing computing intensive research, as well as providing educational opportunities for students and faculty.

- The award is directly responsible for LU's participation in the distributed computing system of the ATLAS High Energy Physics experiment taking place at the Large Hadron Collider at CERN in Geneva, Switzerland. 


\section{Main Success Drivers}

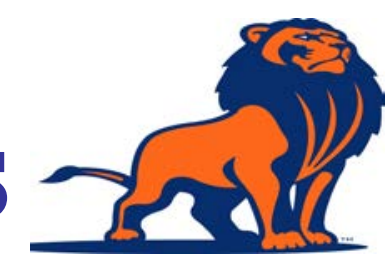

- A major factor in the award was the availability of $\mathbf{1 0}$ Gbps Ethernet (10GE) connectivity for the MRI HPC cluster, Lucille, through Oklahoma's 2010-13 NSF EPSCoR RII C2 grant.

- Thus, OneOCII formed the nucleus of an informal collaborative body of research CI service providers, serving as an informal and ad-hoc incubator of research CI projects. 


\section{Motivators}

SC11 conferences, which were attended by three LU faculty, including MRI Co-PI F. Fondjo Fotou.

- Conversations with other Oklahoma CI leads about the intricacies of developing successful CI MRI projects.

- OneOCII and Oklahoma EPSCoR leadership encouraged one of LU's CI leads to attend an NSF EPSCoR CI meeting in the Washington DC area in 2010.

- A related experience was PI Snow's participation in OU's 2003 CI MRI. 


\section{Key Factors}

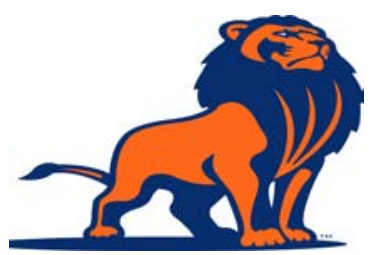

- LU would not only have been underprepared to submit such a proposal without these influences drivers and motivations.

- This was LU's first, and first successful, CI MRI, and it was fully funded at the requested amount $(\$ 250,000)$.

- This MRI funding was the precursor to founding the LU Computing Center for Research and Education (LUCCRE).

- LU intends to submit a follow-up MRI proposal for the next generation system in 2016 or 2017. 
Oklahoma

Innovation

Institute/

Tandy

Superc omputing

Center 


\section{OK Innovation Institute}

n Oklahoma Innovation Institute

- 501(c)(3) not-for-profit corporation supporting economic development in Tulsa and Oklahoma

- Board of Directors includes venture capital professionals and presidents of OU-Tulsa, OSU-Tulsa, U. Tulsa, and Tulsa Community College

- Promotes research collaboration and technology transfer among Tulsa higher education institutions

- Tulsa Regional STEM Alliance

- 60+ STEM workforce and education stakeholders: K-12, afterschool, employers, higher education, professional societies, tribal governments 


\section{ONO OII: Tandy Supercomp Ctr}

Tandy Supercomputing Center

- Shared supercomputing center to broadly serve the Tulsa community

- Primarily academic; commercial access available

- Provide and support education, workforce development, and a "Community Supercomputer" resource

- Funding

- Lead gift from the A.R. \& Marylouise Tandy Foundation

- EDA \#080104715, “Tulsa Community Supercomputer” 


\section{OII and OneOCII}

- OII is not a traditional academic institution:

- As a not-for-profit: OFFN would not have been possible without an academic institution as the lead.

- As a higher education collaboration, OneOCII provides valuable points of contact to OU and OSU's main campuses.

- Tandy Supercomputing Center

- Valuable assistance in system design and general support contributed to the success of OII's Economic Development Administration matching grant. 


\section{OII and OneOCII}

- Tulsa Regional STEM Alliance

- OneOCII provided a powerful joint letter of support from multiple institutions for OII's US2020 City Competition (STEM mentorship).

- Tulsa was among 13 finalists from a field of 52 and ultimately designated "US2020 City Network Founding Partner” -- in-kind support and 2 AmeriCorps VISTAs. 


\section{University of Central Oklahoma}




\section{U Central Oklahoma}

- PUI \& Metropolitan Univ. (OKC Metro)

- 17,000 total students

- National Undergraduate Research Model (CUR)

- Hosting 2018 NCUR Conference!!

- Centers - CURE-STEM, CIBER, CREIC

- Internal (RCSA+Faculty) \& External Grants (NIH/INBRE+NSF)

- UCO needs for HPC resources

- Former Dean Barthell - support for first HPC center

- 2012 established UCO’s Center for Research and Education in Interdisciplinary Computation ( $\underline{\text { CREIC) }}$ 


\section{OneOCII Participation}

- 2004 - NCSI Parallel Computing Workshop at OU (2 faculty)

- 2004 - now Oklahoma SC Symposium (multiple faculty)

- Oklahoma Supercomputing Symposium presentations: 2006, 2008, 2009, 2010, 2014

- SC 2011, 2012, 2014: one or more faculty

- Virtual School for Computational Science \& Engineering: Scientific Visualization workshop remote site at OSU

- OU National Computational Science Institute Parallel Computing workshops

- 2004: 2 faculty

- 20122 faculty 


\section{OneOCII Contribution}

- Participation in OneOCII

- From June 2013

- NSF-MRI support

- Weekly calls

- Comments/suggestions on proposal

- HW on computing needs

- Three other OK institutions with NSF MRI CI recently

- "Acquisition of a High Performance Computing Cluster for Research at a Predominantly Undergraduate Institution,” NSF ACI-1429702, \$304,375, 2015-17, PI E. Lemley

- 4 Attempts! UCO’s first cluster. (OneOCII only on last) 


\section{Buddy!}

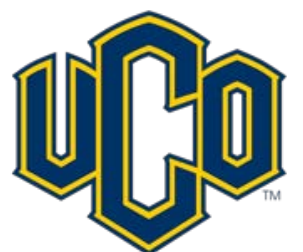

- Helping computational research get done

- NSF - MRI funded recently!

- Buddy Supercomputing Cluster Delivered in June!!
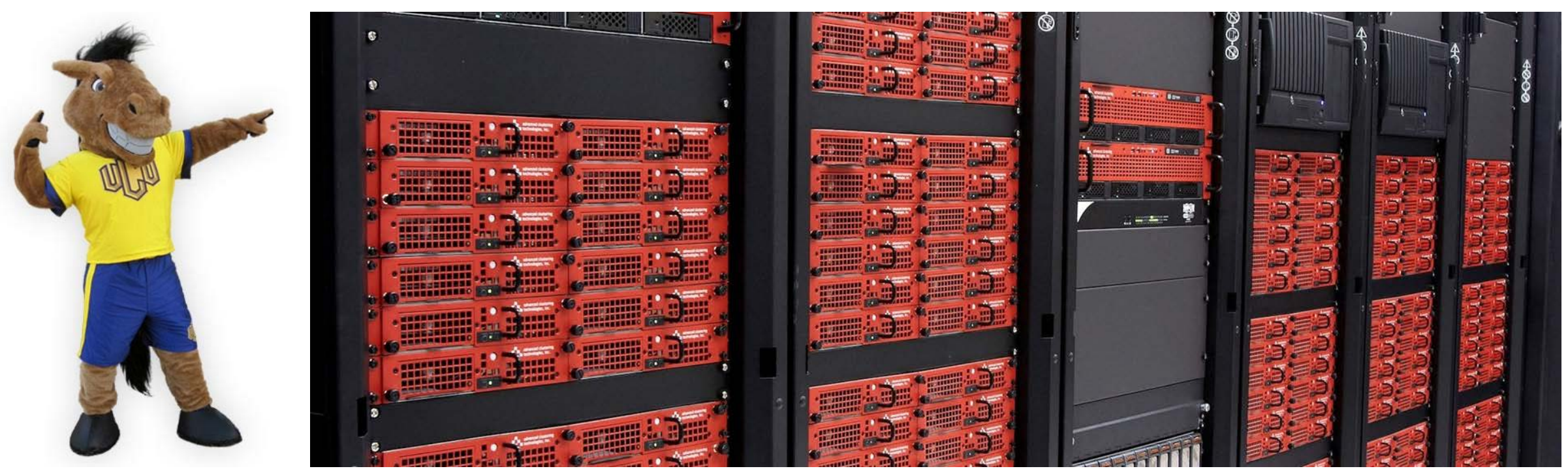

- 30 TFLOPs! ---- bioinformatics, biomechanics, biomedical imaging, microfluidics, phylogenetics, statistics, computational biology, liquid crystals, mathematical biology, intelligent systems, aerodynamics, bioheat transfer, operations research

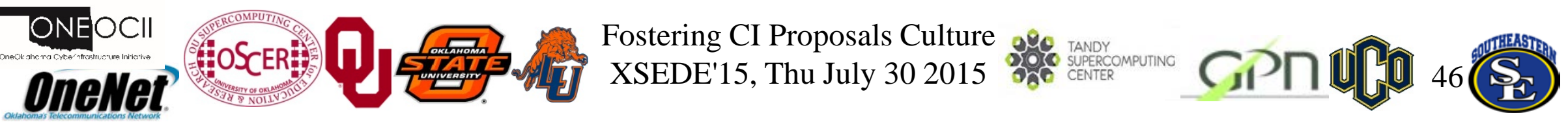


Great Plains Network
and
OneNet 


\section{Great Plains Network}

- Regional CI \& Networking Consortium (4 Oklahoma university members)

- Supercomputing Symposium Academic Sponsor since 2003

- OneOCII Participant

- Interested in seeing the model propagated across the region

- OneOCII shared GPN SC14 exhibit space along with 3 universities

- GPN ENCITE Project (ENabling CyberInfrastructure via Training \& Engagement)

- Funded by NSF Award to Kansas State University

- Co-PIs include OneNet

- Cooperative arrangement with OneOCII for facilitating infrastructure deployment, education and training 


\section{Southeastern Oklahoma State}

University 


\section{Southeastern OK State}

- Regional Liberal Arts University in Durant, OK

- Primarily Undergraduate, with a few Masters Programs

- $30 \%$ of students are Native American

- Approximately 3900 enrolled students Fall 2014

- 235 Faculty (104 have PhDs) 


\section{SE Players}

- Mike Morris

- CS Faculty

- Started attending OU Supercomputing Symposiums in 2005

- Started building compute clusters with students early on

- Applied for LittleFE in 2011

- Karl Frinkle

- Math Faculty

- Recruited by Mike Morris in 2011

- Helped build and install LittleFE on SE campus 2011 


\section{Integrating into OK HPC}

- Teaching Course

- LittleFE instrumental in teaching courses starting in 2011

- One HPC course taught each semester since 2011

- Small cluster build in 2014

- Involvement in the state's HPC community

- Getting in on OneOCII calls

- Attending, presenting at annual OU Supercomputing Symposium

- Booths at Women in Science conference with other OneOCII members 


\section{Grant Opportunity}

\section{Sic}

- GPN ENCITE/Internet2 Broadening the Reach workshop at OU, February 2015

- Discussion about CC-DNI program

- Suggested that SE should write a grant proposal

- Quick deadline, no grant writing experience on SE players

- Mike and Karl agreed, hesitantly 


\section{Proposal Writing}

- Very few proposal writing personnel on SE campus

- No NSF grant proposals had been written for 5 years

- Coordinated with our grant coordinator

- Attended ENCITE/Internet2 grant writing seminar at OU tailored specifically for this grant

- Teleconferenced with multiple OneOCII members

- Many OneOCII members offered guidance on what information needed to be on each part of the grant, and templates were shared with us

- Charlie Peck of Earlham College, current awardee, did teleconference to discuss successful strategy 


\section{Proposal Writing}

- Google Docs was used to help share our work with the proposal with other OneOCII members

- Submission was done on time, one day before deadline

- Would not have been possible without the help of the OneOCII network

- Should know within the month if our grant proposal was accepted 


\section{Recent Papers}

- H. Neeman, K. Adams, J. Alexander, D. Brunson, S. P. Calhoun, J. Deaton, F. Fondjo Fotou, K. Frinkle, Z. Gray, E. Lemley, G. Louthan, G. Monaco, M. Morris, J. Snow and B. Zimmerman, "On Fostering a Culture of Research Cyberinfrastructure Grant Proposals within a Community of Service Providers in an EPSCoR State.” Proc. XSEDE2015.

- H. Neeman, D. Akin, J. Alexander, D. Brunson, S. P. Calhoun, J. Deaton, F. Fondjo Fotou, B. George, D. Gentis, Z. Gray, E. Huebsch, G. Louthan, M. Runion, J. Snow and B. Zimmerman, 2014: "The Fostering CI Proposals Culture: Towards a MultiInstitutional Science DMZ in an EPSCoR State.” Proc. XSEDE2014.

- S. P. Calhoun, D. Akin, J. Alexander, B. Zimmerman, F. Keller, B. George and H. Neeman, 2014: "The Oklahoma PetaStore: A Business Model for Big Data on a Small Budget.” Proc. XSEDE2014.

- C. Carley, B. McKinney, L. Sells, C. Zhao and H. Neeman, 2013: "Using a Shared, Remote Cluster for Teaching HPC.” Proc. IEEE Cluster 2013.

- H. Neeman, D. Brunson, J. Deaton, Z. Gray, E. Huebsch, D. Gentis and D. Horton, 2013: “The Oklahoma Cyberinfrastructure Initiative.” Proc. XSEDE 2013. 


\section{Acknowledgements}

- Portions of this material are based upon work supported by the National Science Foundation under the following grants:

- Grant No. EPS-0814361, "Building Oklahoma's Leadership Role in Cellulosic Bioenergy”

- Grant No. EPS-0919466, “A cyberCommons for Ecological Forecasting”

- Grant No. EPS-1006919, “Oklahoma Optical Initiative”

- Grant No. OCI-10310029, “MRI: Acquisition of Extensible Petascale Storage for Data Intensive Research"

- Grant No. OCI-1126330, “Acquisition of a High Performance Compute Cluster for Multidisciplinary Research”

- Grant No. ACI- 1229107, “Acquisition of a High Performance Computing Cluster for Research and Education”

- Grant No. EPS-1301789, “Adapting Socio-ecological Systems to Increased Climate Variability"

- Grant No. ACI-1341028, “OneOklahoma Friction Free Network”

- Grant No. ACI-1440783, “A Model for Advanced Cyberinfrastructure Research and Education Facilitators”

- Grant No. ACI-1440774, "ENabling CyberInfrastructure via Training and Engagement”

- Dell provided seed systems for the OU Research Cloud (“OURcloud”) and the OU Science DMZ. 


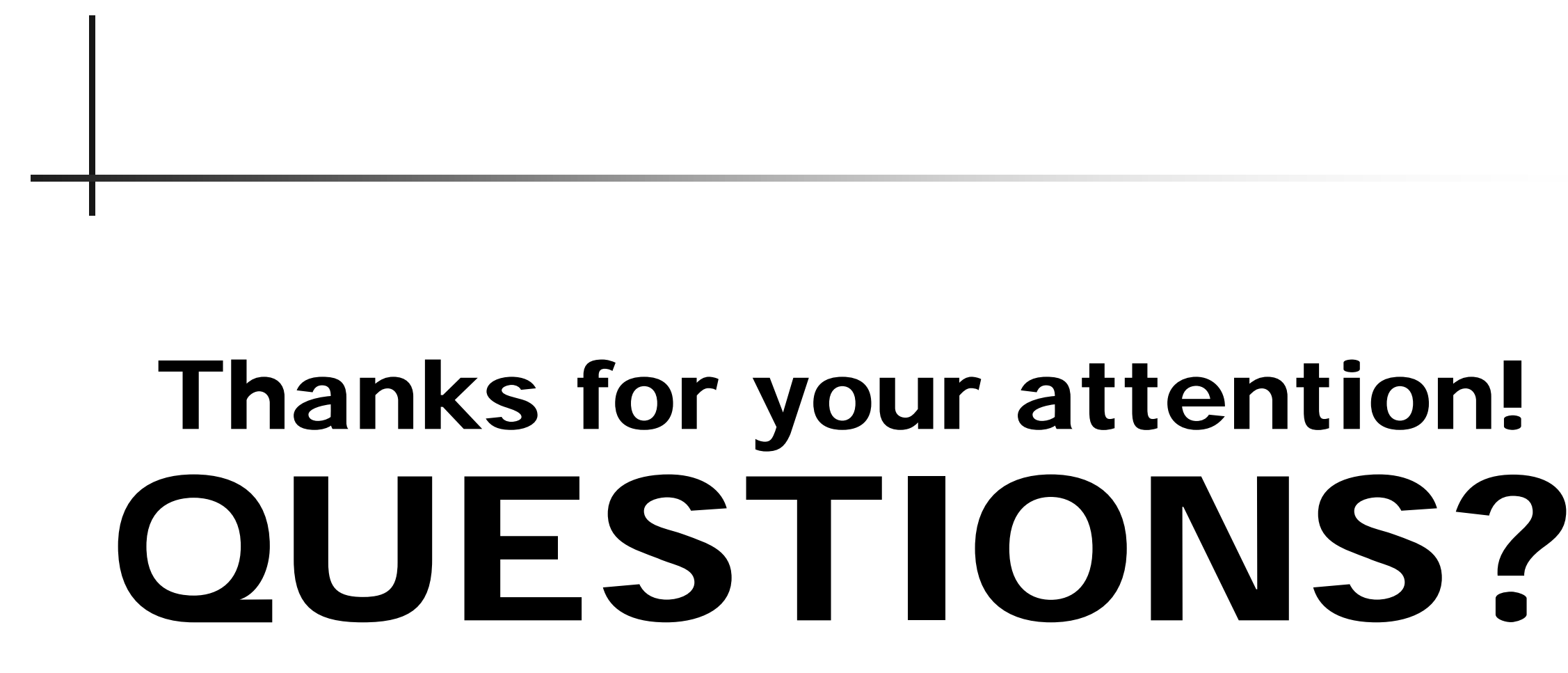

